\title{
Human and entomological surveillance of Toscana virus in the Emilia-Romagna region, Italy, 2010 to 2012
}

\author{
M Calzolari (mattia.calzolari@izsler.it) ${ }^{1}$, P Angelini ${ }^{2}$, A C Finarelli ${ }^{2}$, R Cagarelli ${ }^{2}$, R Bellini ${ }^{3}$, A Albieri ${ }^{3}$, P Bonilauri ${ }^{1}$, F Cavrini ${ }^{4}$,

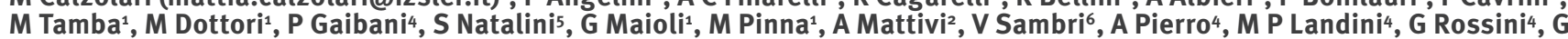 \\ Squintani ${ }^{5}$, S Cinotti ${ }^{1}$, S Varani ${ }^{4}$, C Vocale $^{4}$, E Bedeschi ${ }^{2}$ \\ 1. Istituto Zooprofilattico Sperimentale della Lombardia e dell’Emilia-Romagna, Brescia, Italy \\ 2. Public Health Service, Emilia-Romagna Region, Bologna, Italy \\ 3. Centro Agricoltura Ambiente 'G Nicoli', Crevalcore, Italy \\ 4. Unit of Clinical Microbiology, Regional Reference Centre for Microbiological Emergencies (CRREM), St. Orsola-Malpighi \\ University Hospital, Bologna, Italy \\ 5. Veterinary and Food Hygiene Service, Emilia-Romagna Region, Bologna, Italy \\ 6. Unit of Microbiology, Greater Romagna Area Hub Laboratory, Pievesestina, Italy
}

Toscana virus (TOSV), transmitted by phlebotomine sandflies, is recognised as one of the most important causes of viral meningitis in summer in Mediterranean countries. A surveillance plan based on both human and entomological surveys was started in 2010 in the Emilia-Romagna region, Italy. Clinical samples from patients with neurological manifestations were collected during 2010 to 2012. The surveillance protocol was improved during these years, allowing the detection of 65 human infections. Most of these infections were recorded in hilly areas, where sandflies reach the highest density. Entomological sampling around the homes of the patients resulted in a low number of captured sandflies, while later sampling in a hilly area with high number of human cases $(n=21)$ resulted in a larger number of captured sandflies. Using this approach, 25,653 sandflies were sampled, of which there were 21,157 females, which were sorted into 287 pools. TOSV RNA was detected by real-time PCR in 33 of the pools. The results highlighted the role of Phlebotomus perfiliewi as the main vector of TOSV and a potential link between vector density and virus circulation. This integrated system shows that an interdisciplinary approach improves the sensitiveness and effectiveness of health surveillance.

\section{Introduction}

Toscana virus (TOSV) (family Bunyaviridae, genus Phlebovirus), first isolated from phlebotomine sandflies sampled in the eponymous Italian region in 1971 $[1,2]$, was associated with human disease more than 15 years later $[3,4]$. TOSV is considered not only the causative agent of a self-limiting syndrome, such as sandfly fever (caused by TOSV in Sicily and Naples) [5], characterised by influenza-like symptoms, but also of neurological diseases ranging from aseptic meningitis to meningoencephalitis $[4,6]$.
TOSV is transmitted by some sandflies species, particularly Phlebotomus perfiliewi Parrot, 1911, and Ph. perniciosus Newstead, $1930[1,6]$. In experimental studies, vertical transmission in vectors was reported at rates over $40 \%$ in the first generation after infection $[7,8]$, and has been suggested as a possible mechanism of environmental persistence of the virus $[6,9,10]$. The reservoir role of vertebrates is conceivable, but has been poorly investigated. TOSV circulates during the summer in several Mediterranean countries and was reported as one of the most important causative agents of viral meningitis since the 90 in central Italy $[11,12]$ and since 2000 in France and Spain [9]. Nevertheless, TOSV ecology is still largely unknown: affected sandfly species are not defined and it is unclear whether a vertebrate reservoir exists $[2,6,9]$.

Since the 80s, TOSV circulation has been increasingly reported in the Mediterranean basin, in Portugal, France, Spain, Greece, Bosnia-Herzegovina, Kosovo under UN Security Council Resolution 1244, Malta, Cyprus, Turkey $[6,10,13]$, and since 2012 in Croatia, Morocco and Tunisia [14-16]. In Italy, the virus has been recognised as an agent of meningitis and has been detected in several regions: Tuscany, Piedmont, Marche, Umbria, Lazio, Campania and Sardinia [13]. Since 1999, autochthonous human cases of TOSV meningitis have been reported in the Emilia-Romagna region, indicating circulation of the virus $[17,18]$. In 2010, a surveillance plan to monitor the circulation of TOSV in the Emilia-Romagna region, based on the diagnosis and reporting of human cases and monitoring of vectors, was adopted and gradually improved over the years. Characteristics and data obtained from this surveillance during 2010 to 2012 are described here. 


\section{Methods}

Surveillance of human cases

Human cases of TOSV infection were identified through the regional West Nile virus (WNV) surveillance system that collects samples from suspected cases (blood, serum, cerebrospinal fluid), i.e. hospitalised patients who presented with high fever $\left(38.5^{\circ} \mathrm{C}\right)$ and neuroinvasive manifestations (e.g. encephalitis, meningitis, flaccid paralysis) or polyradiculoneuritis $[19,20]$; patients with febrile illness only were not included in this analysis. According to the regional WNV surveillance guidelines [21], the notification of every suspected case of WNV infection to the public health department is mandatory during the surveillance period, i.e. from June to November.

The diagnostic procedure for identification of TOSV infection has been improved over the years: in 2010, clinical samples collected in July to October for WNV surveillance were retrospectively tested for TOSV [17]; in 2011 and 2012, all WNV surveillance samples were simultaneously tested also for TOSV. Microbiological diagnosis for WNV and TOSV infection was performed by the Regional Reference Centre for Microbiological Emergencies (CRREM) of the Unit of Clinical Microbiology at the St Orsola-Malpighi University Hospital in Bologna, Italy.

On the basis of laboratory findings, TOSV infections were classified as acute (positive PCR with or without IgM detection) or recent (IgM detection alone).

In order to get information about potential places of exposure, home location (urban/rural and hilly/plain), leisure-time habits and perception of the presence of the vector, each TOSV-positive patient was investigated using a standardised WNV surveillance form, administered via telephone by local public health units.

\section{Surveillance of sandflies}

Entomological surveillance was conducted using carbon dioxide-baited traps operating overnight in

\section{FIGURE 1}

Location of human cases of Toscana virus infection by year, in patients resident in the Emilia-Romagna region, Italy ( $\mathrm{n}=61$ )

$0 \quad 10 \quad 20 \quad 30 \quad 40 \quad 50 \mathrm{~km}$

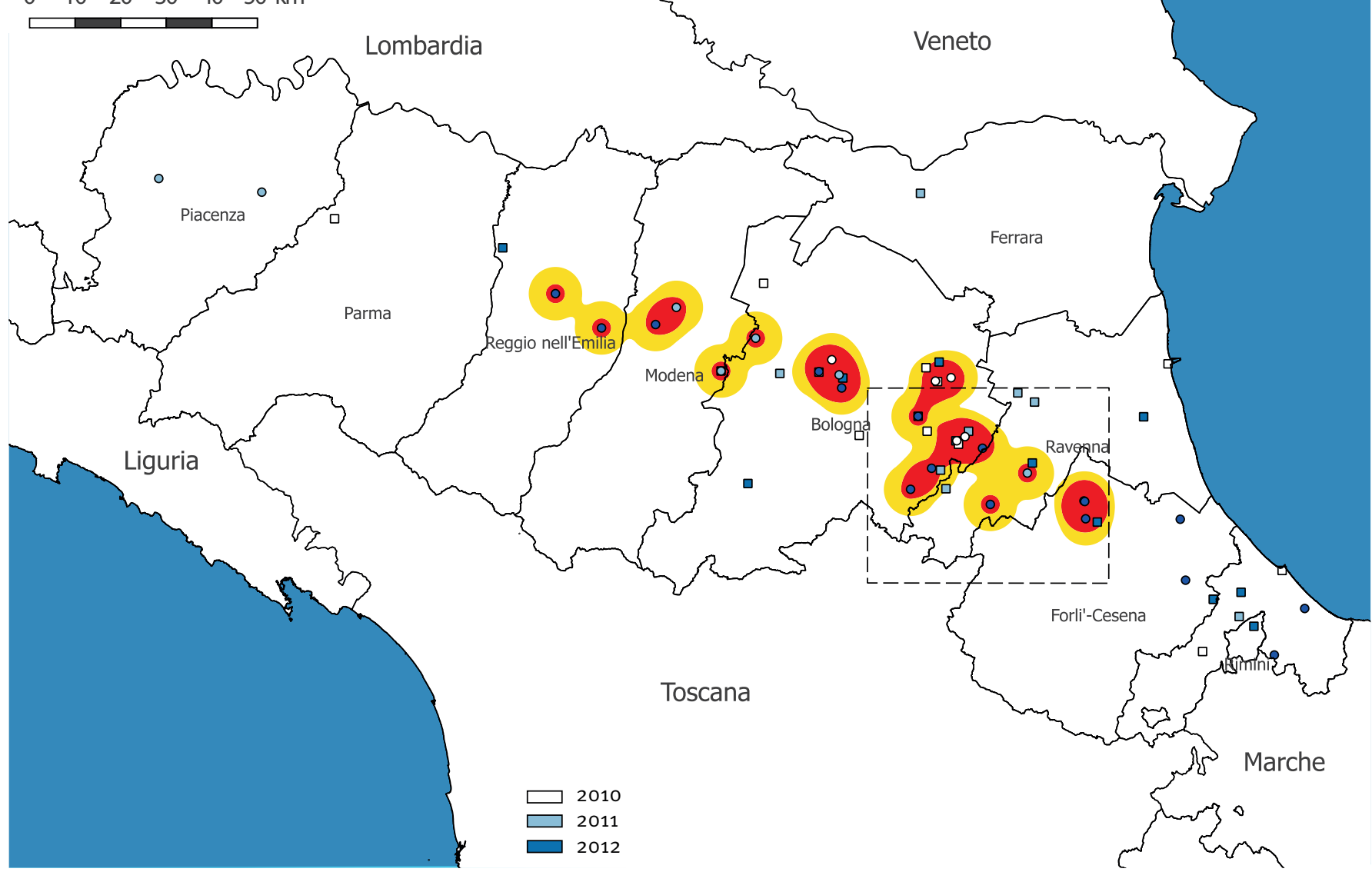

The cases classified as autochthonous $(n=29)$, i.e. they reported to have not travelled or spent nights away from home in the 15 days before symptom onset, used for the percentage volume kernel density estimation (red and yellow areas) are represented by a circle. The large square with the dashed line represents the area of entomological surveillance in 2012, which is shown in Figure 2. 
Sites sampled for Toscana virus in sandflies in 2012 in the Emilia-Romagna region, Italy

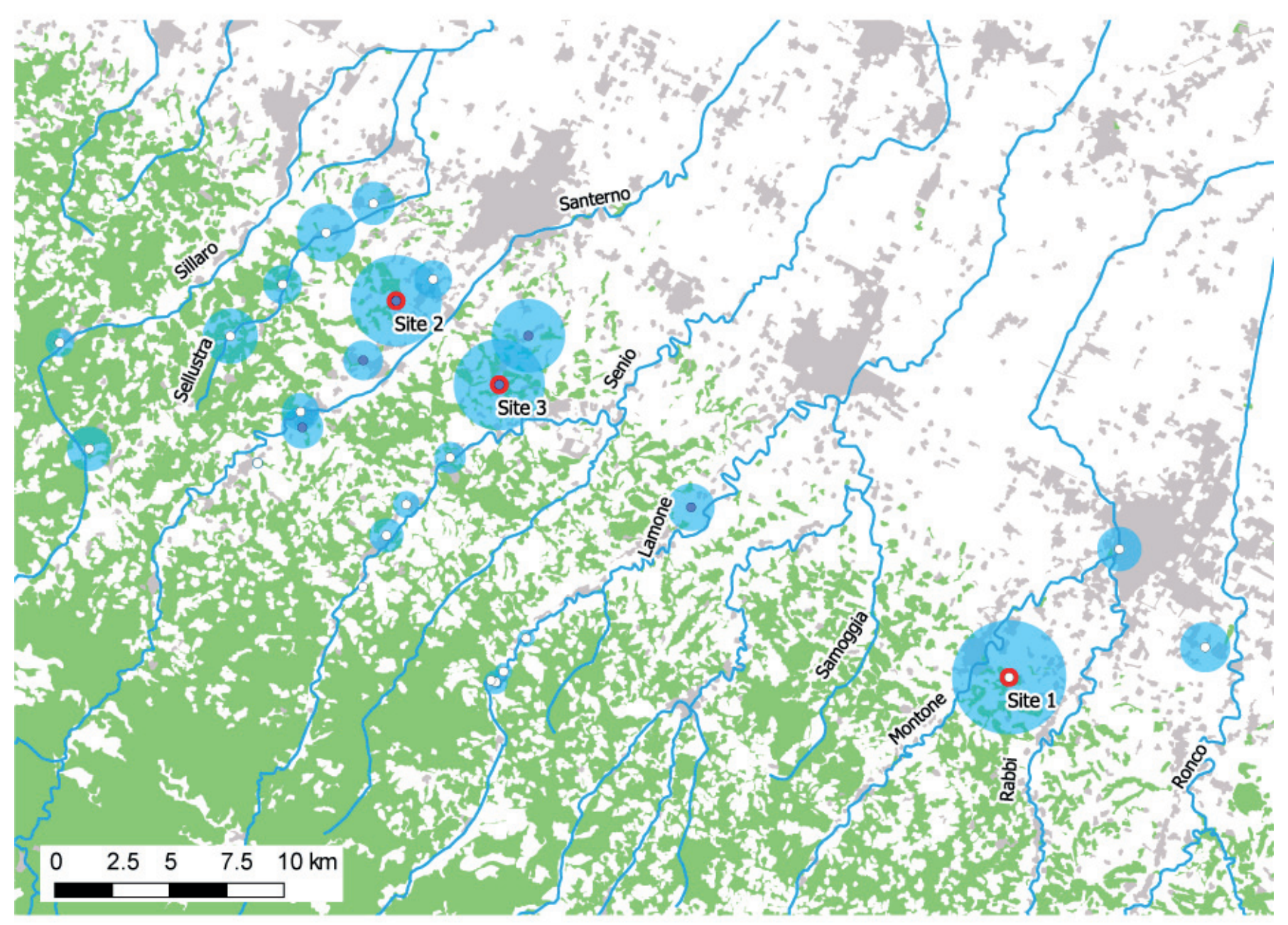

The size of the azure circles is proportional to the number of sandflies collected in a site. Toscana virus-positive pooled female sandflies (red circles), the presence of Phlebotomus perniciosus-positive specimens (blue circles), urban areas (grey), and forest and semi-natural areas (green) are displayed.

The geographical location of the area is shown in Figure 1.

georeferenced sites in the summer, when adult sandfly vectors are present.

In 2010 and 2011, insects were collected for one night from a $200 \mathrm{~m}$ radius around each home (the supposed place of infection) of autochthonous human cases. In 2012, a different strategy was adopted. An area with a high incidence of reported cases was inferred by geographic information system (GIS) analysis: sandflies were collected in this area of about $870 \mathrm{~km} 2$ located in the hilly part of the region (Figure 1). A total of 25 sample sites were selected along the altitude gradient of different river valleys (from west to east: Sillaro, Sellustra, Santerno, Senio, Lamone, Montone and Ronco river valleys) (Figure 2); these sites were sampled twice during June to September. This area has a population density of about 258 inhabitants/km2 [22] and is characterised by cultivated fields intersected with hedges, badlands and woodlands, in which oaks, hornbeams and maples dominate. In this area, the warm temperate climate, characteristic of the region, according to Köppen-Geiger climate classification [23], is mitigated by the proximity of the Adriatic Sea.

If the number of sampled sandflies from a particular location was under 20, all specimens were identified at species level and not submitted to biomolecular analysis.

For samples with more than 20 specimens, the sandflies were divided into groups, up to a maximum of 100 per group, and then the males and females were separated according to the presence of male genitalia. All the males were morphologically identified; pooled females were ground in a $1.5 \mathrm{~mL}$ vial with $500 \mu \mathrm{L}$ of phosphate-buffered saline using a pellet pestle, and then submitted for biomolecular analysis. 
Distribution of human cases of Toscana virus infection resident in the Emilia-Romagna region, Italy, by date of symptom onset, $2010-12(n=61)$

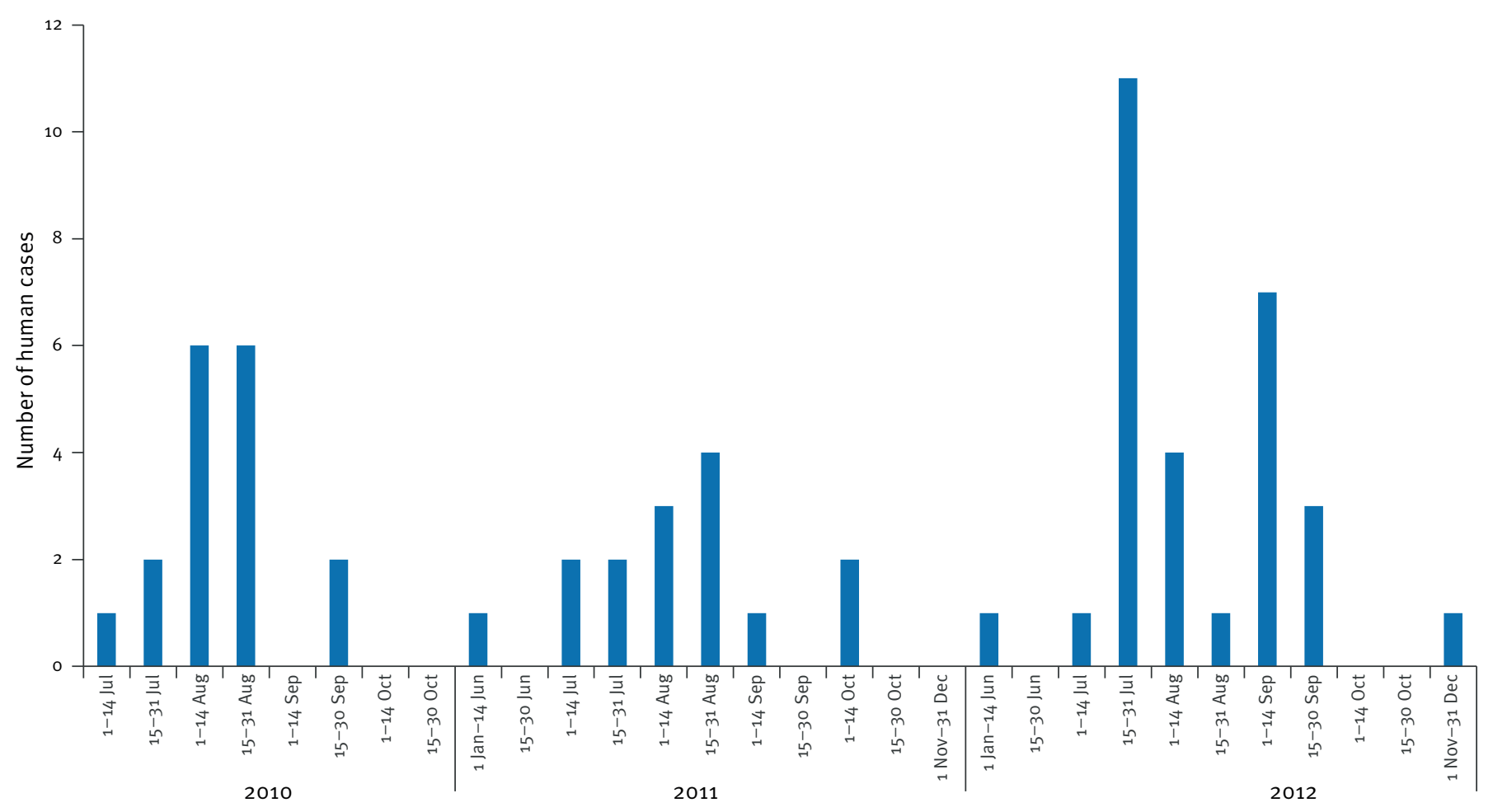

Surveillance period

Morphological identification was performed according to identification keys $[24,25]$. Specimens were observed under an optical microscope after chlorolactophenol clarification to detect morphological characteristics, particularly the shape of the aedeagus, (part of male genitalia) for males, and features of spermathecae and pharynx for females.

\section{Virological analysis}

Laboratory confirmation of human cases of TOSV infection involves molecular and serological testing. We tested for the presence of TOSV RNA in cerebrospinal fluid specimens using a real-time reverse transcription polymerase chain reaction (RT-PCR) for TOSV, targeting the TOSV N gene [26] while anti-TOSV IgG and IgM were detected in serum or plasma samples using an indirect immunofluorescence assay (Euroimmun, Lübeck, Germany). TOSV infections that were classified as acute or recent, as described above, were considered confirmed cases.

Sandfly RNA was extracted using TrizolLS Reagent (Invitrogen, Carlsbad, CA, United States); cDNA synthesis was achieved using random hexamers (Roche Diagnostics, Mannheim, Germany) and SuperScriptH II reverse transcriptase (Invitrogen, Carlsbad, CA) and then tested by a real time RT-PCR specific for TOSV detection [26].

\section{Statistical and GIS analysis}

Autochthonous human cases of TOSV infection from 2010 to 2012 were geocoded from the Public Health database. The pattern of spatial data was characterised by conducting nearest neighbour analysis on the locations of the human cases of TOSV infection. Analyses were performed using surveillance information on 60 TOSV-positive patients identified; such data were not available for one case.

Kernel density estimation was applied to the location of the autochthonous human cases, in order to assess TOSV cluster presence in the Emilia-Romagna region. This geospatial technique, based on a kernel function (a quadratic function in this analysis), was used to create a surface to indicate the intensity of a particular event; the mean distance between the autochthonous cases $(8 \mathrm{~km})$ was chosen as bandwidth. The cluster area of TOSV presence was estimated by the percentage volume contour of the TOSV kernel density estimation, which represents the boundary of the area containing $95 \%$ and $50 \%$ of the volume of the obtained kernel density estimation. Nearest neighbour analysis and kernel density estimation were performed using ESRI ArcGIS 9.3 and the Spatial Analyst extension.

To test whether the risk of TOSV infection is related to a patient's sex, a general linear model was applied and logistic regression used on the dataset of tested patients using the software Intercooled Stata 7.0 
(probability of infection was the dependent variable and sex was the independent variable).

Maximum likelihood estimation of infected specimens for 1,000 female sandflies collected, for every dataset and sampled sites, was obtained using the United States Centers for Disease Control and Prevention pool infection rate Excel add-in version 4.0 [27].

\section{Results}

\section{Human cases}

During 2010 to 2012, samples obtained from 380 patients were screened within the framework of the regional surveillance system (120 in 2010, 140 in 2011 and 120 in 2012). Of these, 152 were female (62 in 2010, 45 in 2011, and 45 in 2012) and 228 were males (58 in 2010, 95 in 2011, and 75 in 2012).

TOSV infection was confirmed in 65 patients; of these, 61 (17 in 2010, 15 in 2011, and 29 in 2012) probably were infected inside the Emilia-Romagna region; the additional four laboratory-confirmed cases were excluded from our analysis because the patients were not resident in the Emilia-Romagna region and therefore were probably exposed to the infection elsewhere (two patients were from Tuscany, one from Marche and one from San Marino). In 2010 and 2011, the peak of cases was recorded in August, while in 2012 the maximum number of cases was detected at the end of July, with a possible second peak at the beginning of September (Figure 3).
Among the infected patients, a consistently high proportion of men was observed each year (44/61 in the whole period; $11 / 17$ cases in 2010, $11 / 15$ in 2011, 22/29 in 2012). In the whole period, the probability of tested patients being infected by the virus was 0.24 (95\% Cl: $0.18-0.30$ ) for male patients and 0.13 (95\% Cl: $0.8-$ 0.18) for female patients. Thus, tested patients infected with TOSV were about twice as likely to be male (odds ratio: 1.90 (95\% Cl: 1.04-3.47); p<0.05).

The median age of the TOSV-infected patients analysed was 41 years (range: 16-83); 29/61 were aged 25-44 years. The most frequent manifestation in the patients was meningitis $(n=40)$. Of the 61 confirmed cases, 43 were classified as acute infections, according to positive PCR or both positive PCR and IgM detection (Table 1). Additional information on the surveillance form (available for 60 cases) indicated that 29 patients reported to have not travelled or spent nights away from home in the 15 days before symptom onset. So it is hypothesised that they acquired the infection nearby their home: these cases were classified as autochthonous cases. The place of residence of these cases was used in the kernel density estimation, which indicated that the areas at higher risk of TOSV circulation were located in the hilly part of the region (Figure 1 ).

Furthermore, 16 of the these 60 cases declared a perception of a high density of biting insects in the area

\section{TABLE 1}

Demographic and clinical characteristics of human cases of Toscana virus infection resident in the Emilia-Romagna region, Italy, 2010-12 ( $\mathrm{n}=61)$

\begin{tabular}{|c|c|c|c|c|}
\hline \multirow{3}{*}{ Characteristic } & \multicolumn{4}{|c|}{ Year } \\
\hline & 2010 & 2011 & 2012 & $2010-12$ \\
\hline & Number & Number & Number & Number \\
\hline $0-14$ & 0 & 0 & 0 & 0 \\
\hline $15-24$ & 2 & 2 & 2 & 6 \\
\hline $45-64$ & 4 & 4 & 6 & 14 \\
\hline$\geq 65$ & 3 & 0 & 8 & 11 \\
\hline \multicolumn{5}{|l|}{ Sex } \\
\hline Male & 11 & 11 & 22 & 44 \\
\hline Female & 6 & 4 & 7 & 17 \\
\hline \multicolumn{5}{|l|}{ Clinical features } \\
\hline Meningitis & 13 & 11 & 16 & 40 \\
\hline Meningoencephalitis & 0 & 1 & 7 & 8 \\
\hline \multicolumn{5}{|l|}{ Type of infection } \\
\hline Acute infection $^{a}$ & 5 & 11 & 27 & 43 \\
\hline Recent infection ${ }^{b}$ & 12 & 4 & 2 & 18 \\
\hline Total & 17 & 15 & 29 & 61 \\
\hline
\end{tabular}

a Positive PCR [26] with or without IgM detection.

b Only positive IgM detection. 
TABLE 2

Sandflies sampled in 2012 by river valley of collection, Emilia-Romagna region, Italy

\begin{tabular}{|l|c|c|c|c|c|}
\hline River valley & Number of sites & $\begin{array}{c}\text { Altitude range } \\
\text { (metres above sea } \\
\text { level) }\end{array}$ & $\begin{array}{c}\text { Number of collected } \\
\text { sandflies }\end{array}$ & $\begin{array}{c}\text { Number female } \\
\text { Number male (P.pf/P. } \\
\text { pn) }\end{array}$ \\
\hline Sillaro & 2 & $191-341$ & 252 & 157 & $95(95 / 0)$ \\
\hline Sellustra & 4 & $133-226$ & 1,071 & 842 & $229(229 / 0)$ \\
\hline Santerno & 6 & $78-175$ & 5,305 & 3,655 & $1,650(1,645 / 5)$ \\
\hline Senio & $6^{\mathrm{a}}$ & $130-207$ & 6,733 & 5,139 & $1,594(1,589 / 5)$ \\
\hline Lamone & $5^{\mathrm{b}}$ & $83-232$ & 316 & 285 & $31(30 / 1)$ \\
\hline Montone & 2 & $27-183$ & 11,565 & 10,746 & $819(819 / 0)$ \\
\hline Ronco & 1 & 32 & 411 & 333 & $78(78 / 0)$ \\
\hline Total & 26 & $27-341$ & 25,653 & 21,157 & $4,496(4,485 / 11)$ \\
\hline
\end{tabular}

P.pf: Phlebotomus perfiliewi; P.pn: Phelbotomus perniciosus.

a Three sites with no sandflies captured.

${ }^{\mathrm{b}}$ One site with no sandflies captured.

around their home and 40 used personal or home protection measures against biting insects.

\section{Entomological findings}

In 2010 and 2011, sandflies were collected in 15 sites in areas around the homes of human cases (nine in 2010 and six in 2011). This approach resulted in a small number of sandflies being captured; in seven of the sites, no sandflies were collected. In 2010, a total of 54 sandflies were collected from four sites; in 2011, a total of 200 specimens were captured in four sites (of these, 197 were collected in one site).

All sandflies identified in 2010 belonged to $\mathrm{Ph}$. perfiliewi species; in 2011, 110 specimens were identified: two were $P h$. perniciosus (in one site), while the other sandflies were identified as $\mathrm{Ph}$. perfiliewi.

Due to the low number of sampled specimens, only two pools underwent biomolecular analysis, which gave negative results.
The change of strategy in 2012 greatly increased the number of sampled sandflies, leading to the detection of TOSV-infected vectors. A total of 26 selected sites, ranging in altitude from $27 \mathrm{~m}$ to $341 \mathrm{~m}$ above sea level were sampled twice in the season, about a month apart. From 26 July to 28 September, a total of 25,653 sandflies $(21,157$ female and 4,496 male) were captured in 22 of these sites (Table 2). The females were sorted into 287 pools, of which 33 tested positive for TOSV (Table 3).

The 33 TOSV-positive pools were from three sites, with the altitude of the positive sites ranging from $128 \mathrm{~m}$ to $207 \mathrm{~m}$ above sea level. At one of the sites (Site 2), TOSV-positive pools were detected on two different sampling days. The maximum likelihood estimation of each pool varied from 1.5 to 3.8 (Table 3 ).

Of the male sandflies analyses, Ph. perfiliewi was the preponderant species in the sampled areas (only $11 \mathrm{Ph}$. perniciosus males in a total of 4,496 male sandflies).

\section{TABLE 3}

Analysis of samples from sites in which Toscana virus was detected in sandflies, Emilia-Romagna region, Italy, 2012

\begin{tabular}{|c|c|c|c|c|c|c|c|c|}
\hline Site & River & $\begin{array}{c}\text { Altitude } \\
\text { (metres above } \\
\text { sea level) }\end{array}$ & $\begin{array}{c}\text { Date in } \\
2012\end{array}$ & $\begin{array}{l}\text { Number of } \\
\text { sandflies } \\
\text { collected }\end{array}$ & $\begin{array}{l}\text { Number male } \\
\text { (P.pf/P.pn) }\end{array}$ & $\begin{array}{l}\text { Number } \\
\text { female }\end{array}$ & $\begin{array}{l}\text { Number of pools } \\
\text { of females } \\
\text { positive/total }\end{array}$ & $\begin{array}{l}\text { MLE (lower } \\
\text { limit-uppper } \\
\text { limit) }\end{array}$ \\
\hline \multirow{2}{*}{1} & \multirow{2}{*}{ Montone } & \multirow{2}{*}{183} & 2 Aug & 9,295 & 727 (727/0) & 8,568 & $19 / 93$ & $2.5(1.5-3.8)$ \\
\hline & & & 7 Sep & 2,367 & 97 (97/0) & 2,270 & $0 / 24$ & NA \\
\hline \multirow{2}{*}{2} & \multirow{2}{*}{ Santerno } & \multirow{2}{*}{128} & $26 \mathrm{Jul}$ & 2,285 & 494 (494/o) & 1,791 & $6 / 23$ & $3.8(1.6-7.9)$ \\
\hline & & & 30 Aug & 2,400 & $1,075(1073 / 2)$ & 1,325 & $2 / 24$ & $1.5(0.3-4.9)$ \\
\hline \multirow{2}{*}{3} & \multirow{2}{*}{ Senio } & \multirow{2}{*}{207} & 17 Aug & 4,660 & $933(930 / 3)$ & 3,727 & $6 / 48$ & $1.7(0.7-3.5)$ \\
\hline & & & $28 \mathrm{Sep}$ & 10 & $1(1 / 0)$ & 9 & $0 / 2$ & NA \\
\hline Total & - & - & - & 21,017 & $3,327(3,322 / 5)$ & 17,690 & $33 / 214$ & NA \\
\hline
\end{tabular}


All the Ph. perniciosus specimens were found in three neighbouring valleys (Santerno, Senio and Lamone) (Table 2, Figure 2).

\section{Discussion}

The fact that there were no patients under 14 years of age is in line with previous observations [28]. There may be two reasons for the lack of cases of TOSV infection in children: TOSV infection may occur at a lower rate in children or it may be more frequently asymptomatic or paucisymptomatic in children than in adults. A high proportion of adult cases and a larger presence of males among confirmed cases has already been reported in serological investigations in Italy [29] and could be linked to behaviour, with high levels of outdoor activities having been described as a risk factor of TOSV infection [9]. This observation may explain the greater likelihood of infection in males, as highlighted by our surveillance.

The location of most of the human cases (46/61) in hilly areas of the region is an expected result as these areas are the typical habitat for sandflies and support a very high density of vectors, as confirmed by the abundance of sandflies collected (Tables 2 and 3). Two species of sandflies were collected during the survey, Ph. perfiliewi $(4,647 / 4,660 ; 99.7 \%)$ and Ph. perniciosus (13/4,660 0.3\%). Both species are considered efficient vectors of TOSV [6], but due to its abundance, $P h$. perfiliewi seems to be the main vector of TOSV in the Emilia-Romagna region. As the two species have a different distribution in the Mediterranean basin, Ph. perniciosus in western part and $P h$. perfiliewi in eastern part, with an overlap between the two in the central Mediterranean area, [10], confirmation of the vectorial competence of both species enlarges the area of potential TOSV presence. There is, however, a need for precise data on the distribution of sandflies in Europe, data that are often not available [10].

A major role of vertical transmission in the persistence of TOSV in the environment has been hypothesised, but vertical transmission seems ineffective in ensuring such persistence of the virus over generations, at least in experimental studies $[7,8]$. Possible involvement of vertebrates in the TOSV life cycle could be hypothesised as antibodies against the virus have been detected in horses and sheep in Italy [13] and in horses, goats, pigs, cats, dogs, sheep and cows in Spain; in one of the goat samples, the virus was detected by real-time PCR [30]. However, it should be borne in mind that the presence of anti-TOSV antibodies in these animals does not imply that they play a role in the natural cycle of the virus. In a survey conducted in Italy between 1983 and 1985 , one TOSV strain was isolated from a bat captured in 1984, which had tested negative in a serological test [2]. The possible involvement of other vertebrates, such as rodents, in the life cycle of various phleboviruses transmitted by sandflies has been suggested previously [5]. Further studies are needed to identify possible TOSV reservoirs but, considering that sandflies do not fly for long distances [31] and that
TOSV was found in sandflies collected in rural areas, our study indicates that a putative reservoir, if present, could be looked for in such rural environments.

The initial approach of collecting sandflies in areas nearby the homes of the human cases gave poor results, probably due to the time elapsed (estimated to be about two weeks) between the onset of the human infection and the sampling of sandflies. This may have resulted in a reduction in the number of sandflies, as these insects show sharp peaks of abundance during the season and have a short lifespan, of about two or three weeks $[10,25,31]$. The 2012 sampling methodology, which focused on a hilly area with observed TOSV circulation in previous years, irrespective of whether human cases had been detected nearby, resulted in the capture of a greater number of sandflies. Interestingly, in all sampled sites in which TOSV was identified, the virus was detected, or a greater maximum likelihood estimation observed, in early samples, showing the highest circulation of the virus to be between the end of July and the beginning of August (Table 3). This is in agreement with human epidemiological data obtained in 2012, which showed a similar early peak in human cases at the end of July (Figure 3). This may be linked to the exceptional drought conditions recorded in 2012, a year with one of the driest Augusts recorded in the time series of regional meteorological services [32], which date back to 1950s. In 2012, the occurrence of human cases seemed to be different from that of previous years: in 2010-11, the peak of human cases was recorded in August (Figure 3), reflecting the classic dynamic described in the literature $[4,13]$.

The different trends of human cases during the study period may be due to different population dynamics of the vector. This hypothesis seems to be confirmed by the detection of TOSV in the more abundant samples obtained during the 2012 survey, in which more than 18,000 specimens were collected, more than $70 \%$ of the sandflies collected throughout the study period. Moreover, it is thought that sandflies could be both the main reservoir and also have an amplifying role in the life cycle of TOSV $[6,10]$. Previous field evidence has linked the peak in human cases with periods of highest density of the vector [4]. Taken together, these results seem to suggest a strong correlation between the presence of the virus and sandfly density. If confirmed, this correlation could be a very useful tool for assessing the risk of TOSV infection. The importance of these findings is emphasised by different field studies that indicate an increasing in density and a northern spread of sandflies in Europe [33], with a consequent increasing burden of sandfly transmitted diseases $[10,33]$.

The integrated surveillance system we have described was able to identify areas in which TOSV circulation was more intense and the risk of human infection was the highest. Improvements in the surveillance strategy over the years have resulted in the availability of epidemiological data about the TOSV life cycle, which may 
be useful to obtain models to forecast TOSV circulation in the region. If the hypothesis of correlation between vector abundance and virus circulation is confirmed, this correlation could be used to develop effective and efficient actions intended to prevent virus transmission, such as vector monitoring, vector control policies and informative campaigns, to stimulate the adoption of personal protection measures in risk areas.

\section{Conflict of interest}

None declared.

\section{Authors' contributions}

MC: entomology field and laboratory work, data analysis, drafted the article. PA: organisation of surveillance, organisation of surveillance. RC: organisation of surveillance, data analysis. RB: entomology field work, drafted the article. AA: entomology field work, GIS analysis. PB: entomology laboratory work, data analysis. FC: laboratory work on human samples. MD: organisation of surveillance. PG: laboratory work on human samples. SN: organisation of surveillance. GM: laboratory work on entomological samples. MP: laboratory work on entomological samples. AM: data analysis, drafted the article. VS: laboratory work on human samples, drafted the article. AP: laboratory work on human samples, data analysis, drafted the article. MPL: laboratory work on human samples. GR: laboratory work on human samples. GS: organisation of surveillance. SC: organisation of surveillance. SV: laboratory work on human samples. CV: laboratory work on human samples, data analysis, drafted the article. EB: organisation of surveillance.

\section{References}

1. Verani P, Ciufolini MG, Nicoletti L, Balducci M, Sabatinelli G, Coluzzi M, et al. [Ecological and epidemiological studies of Toscana virus, an arbovirus isolated from Phlebotomus]. Ann Ist Super Sanita. 1982;18(3):397-9. Italian. PMID:7187828

2. Verani P, Ciufolini MG, Caciolli S, Renzi A, Nicoletti L, Sabatinelli G, et al. Ecology of viruses isolated from sand flies in Italy and characterized of a new Phlebovirus (Arabia virus). Am J Trop Med Hyg. 1988;38(2):433-9. Available from: PMID:3128131

3. Calisher CH, Weinberg AN, Muth DJ, Lazuick JS. Toscana virus infection in United States citizen returning from Italy. Lancet. 1987;1(8525):165-6. http://dx.doi.org/10.1016/So1406736(87)92005-8 PMID:2880004

4. Nicoletti L, Verani P, Caciolli S, Ciufolini MG, Renzi A, Bartolozzi D, et al. Central nervous system involvement during infection by Phlebovirus toscana of residents in natural foci in central Italy (1977-1988). Am J Trop Med Hyg. 1991;45(4):42934. Available from: PMID:1951851

5. Ashford RW. Phlebotomus fevers. In: Service M. The encyclopedia of arthropod-transmitted Infections. Wallingford: CABI; 2001. p. 397-401.

6. Charrel RN, Gallian P, Navarro-Mari JM, Nicoletti L, Papa A, Sánchez-Seco MP, et al. Emergence of Toscana virus in Europe. Emerg Infect Dis. 2005;11(11):1657-63. http://dx.doi. org/10.3201/eid1111.050869 PMID:16318715

7. Tesh RB, Modi GB. Maintenance of Toscana virus in Phlebotomus perniciosus by vertical transmission. Am J Trop Med Hyg. 1987;36(1):189-93. Available from: PMID:3028194

8. Maroli M, Ciufolini MG, Verani P. Vertical transmission of Toscana virus in the sandfly, Phlebotomus perniciosus, via the second gonotrophic cycle. Med Vet Entomol. 1993;7(3):2836. http://dx.doi.org/10.1111/j.1365-2915.1993.tboo689.x PMID:8369564

9. Charrel RN, Bichaud L, de Lamballerie X. Emergence of Toscana virus in the mediterranean area. World J Virol. 2012;1(5):135-41. PMID:24175218

10. Alkan C, Bichaud L, de Lamballerie X, Alten B, Gould EA Charrel RN. Sandfly-borne phleboviruses of Eurasia and Africa: epidemiology, genetic diversity, geographic range, contro measures. Antiviral Res. 2013;100(1):54-74. http://dx.doi. org/10.1016/j.antiviral.2013.07.005 PMID:23872312

11. Valassina M, Cuppone AM, Bianchi S, Santini L, Cusi MG. Evidence of Toscana virus variants circulating in Tuscany, Italy, during the summers of 1995 to 1997. J Clin Microbiol. 1998;36(7):2103-4. Available from: PMID:9650974

12. Valassina M, Meacci F, Valensin PE, Cusi MG. Detection of neurotropic viruses circulating in Tuscany: the incisive role of Toscana virus. J Med Virol. 2000;60(1):86-90. http:// dx.doi.org/10.1002/(SICI)1096-9071(200001)60:1<86::AIDJMV14>3.0.CO;2-N PMID:10568768

13. Cusi MG, Savellini GG, Zanelli G. Toscana virus epidemiology: from Italy to beyond. Open Virol J. 2010;4:22-8. PMID:20517492

14. Punda-Polić V, Mohar B, Duh D, Bradarić N, Korva M, Fajs L, et al. Evidence of an autochthonous Toscana virus strain in Croatia. J Clin Virol. 2012;55(1):4-7. http://dx.doi.org/10.1016/j. jcv.2012.06.006 PMID:22795597

15. Es-Sette N, Nourlil J, Hamdi S, Mellouki F, Lemrani M. First detection of Toscana virus RNA from sand flies in the genus Phlebotomus (Diptera: Phlebotomidae) naturally infected in Morocco. J Med Entomol. 2012;49(6):1507-9. http://dx.doi. org/10.1603/ME12042 PMID:23270183

16. Bichaud L, Dachraoui K, Piorkowski G, Chelbi I, Moureau G, Cherni $S$, et al. Toscana virus isolated from sandflies, Tunisia. Emerg Infect Dis. 2013;19(2):322-4. http://dx.doi.org/10.3201/ eid1902.121463 PMID:23460990

17. Portolani M, Sabbatini AM, Beretti F, Gennari W, Tamassia MG, Pecorari M. Symptomatic infections by toscana virus in the Modena province in the triennium 1999-2001. New Microbiol. 2002;25(4):485-8. Available from: PMID:12437229

18. Vocale C, Bartoletti M, Rossini G, Macini P, Pascucci MG, Mori F, et al. Toscana virus infections in northern Italy: laboratory and clinical evaluation. Vector Borne Zoonotic Dis. 2012;12(6):526-9. http://dx.doi.org/10.1089/vbz.2011.0781 PMID:22448679

19. Angelini P, Tamba M, Finarelli AC, Bellini R, Albieri A, Bonilauri $P$, et al. West Nile virus circulation in Emilia-Romagna, Italy: the integrated surveillance system 2009. Euro Surveill. 2010;15(16):19547. Available from: PMID:20430000

20. Rizzo C, Salcuni P, Nicoletti L, Ciufolini MG, Russo F, Masala R, et al. Epidemiological surveillance of West Nile neuroinvasive diseases in Italy, 2008 to 2011. Euro Surveill. 2012;17(20):20172. Available from: PMID:22642945

21. Regione Emilia-Romagna. Piano di sorveglianza e controllo Chikungunya e Dengue e malattia West Nile in Emilia-Romagna, anno 2011. [Plan for surveillance and control of Chikungunya, Dengue, and West Nile disease in Emilia-Romagna, 2011]. Bologna: Regione Emilia-Romagna. [Accessed 1 Dec 2014]. Italian. Available from: http://www.saluter.it/documentazione/ piani-e-programmi/chik_dengue_westnile_2011.pdf

22. Servizio statistica e informazione geografica Emilia-Romagna E-R Statistica. [Emilia-Romagna statistics]. Bologna: Servizio statistica e informazione geografica Emilia-Romagna. Italian. Available from: http://statistica.regione.emilia-romagna.it/

23. Kottek M, Grieser J, Beck C, Rudolf B, Rubel F. World map of the Köppen-Geiger climate classification updated. Meteorol. Z. 2006;15(3):259-63. http://dx.doi. org/10.1127/0941-2948/2006/0130

24. Lewis DJ. A taxonomic review of the genus Phlebotomus (Diptera: Psychodidae). Bulletin of the British Museum (Natural History). Entomology Series. 1982;45(2):121-209.

25. Romi R, Khoury C, Bigliocchi F, Maroli M. Schede guida su acari e insetti di interesse sanitario. [Fact sheet on mites and insects of medical importance]. Rome: Istituto Superiore di Sanità; 1994. Italian. http://www.iss.it/binary/publ/cont/ Pag1_20Rapp94_08.pdf

26. Pérez-Ruiz M, Collao X, Navarro-Marí JM, Tenorio A. Reverse transcription, real-time PCR assay for detection of Toscana virus. J Clin Virol. 2007;39(4):276-81. http://dx.doi. org/10.1016/j.jcv.2007.05.003 PMID:17584525

27. Biggerstaff BJ. PooledInfRate, Version 4.0: a Microsoft ${ }^{\circledR}$ Office Excel(C) Add-In to compute prevalence estimates from pooled samples. Fort Collins, CO: United States Centers for Disease Control and Prevention; 2009.

28. Terrosi C, Olivieri R, Bianco C, Cellesi C, Cusi MG. Agedependent seroprevalence of Toscana virus in central Italy and correlation with the clinical profile. Clin Vaccine Immunol. 2009;16(8):1251-2. http://dx.doi.org/10.1128/CVI.00376-08 PMID:19553552

29. D’Ovidio MC, Venturi G, Fiorentini C, Barbati G, Di Renzi $\mathrm{S}$, Vonesch N, et al. Occupational risk associated with Toscana virus infection in Tuscany, Italy. Occup Med (Lond). 2008;58(8):540-4. http://dx.doi.org/10.1093/occmed/kqn131 PMID:18832346 
30. Navarro-Marí JM, Palop-Borrás B, Pérez-Ruiz M, SanbonmatsuGámez S. Serosurvey study of Toscana virus in domestic animals, Granada, Spain. Vector Borne Zoonotic Dis. 2011;11(5):583-7. http://dx.doi.org/10.1089/vbz.2010.0065 PMID:20925529

31. Killick-Kendrick R. The biology and control of phlebotomine sand flies. Clin Dermatol. 1999;17(3):279-89. http://dx.doi. org/10.1016/S0738-081X(99)00046-2 PMID:10384867

32. ARPA. Bollettino agrometeorologico mensile Agosto 2012. [Monthly agrometeorological bulletin]. [Accessed 9 Jun 2014]. Italian. Available from: http://www.arpa.emr.it/

33. Maroli M, Feliciangeli MD, Bichaud L, Charrel RN, Gradoni L. Phlebotomine sandflies and the spreading of leishmaniases and other diseases of public health concern. Med Vet Entomol. 2013;27(2):123-47. http://dx.doi.org/10.1111/j.13652915.2012.01034.x PMID:22924419 